\title{
Técnica fenol alcohol para el tratamiento de uñas encarnadas. Tiempo medio de curación y complicaciones para una muestra de mayores de 65 años
}

\author{
Phenol alcohol technique for treating ingrown toenails. Healing throught \\ time and complications for a sample of over 65
}

\author{
Laura Lasanta Lacalzada ${ }^{1}$, Raúl Reyes Martín ${ }^{2}$ \\ ${ }^{1}$ Diplomado Universitario Enfermería. Diplomado en Podología. Máster de Investigación en Ciencias Podológicas \\ de la Universidad Rey Juan Carlos de Madrid. \\ lauralasanta1@hotmail.com \\ ${ }^{2}$ Diplomado Universitario Podología. Máster de Investigación en Ciencias Podológicas de la Universidad Rey Juan \\ Carlos de Madrid. \\ clinicadelpie@live.com
}

Correspondencia:

Raúl Reyes Martín

Dirección del centro de trabajo:

C/Camino Viejo de Leganés $177-1^{\circ}$, puerta 8

E-28025 Madrid

Correo electrónico: clinicadelpie@live.com

Fecha de recepción: 23 de febrero de 2013

Fecha de aceptación: 22 de mayo de 2013

Los autores declaran no tener ningún tipo de interés económico o comercial.

\section{RESUMEN}

Estudio original.

Introducción. La matricectomía química por fenol alcohol es una técnica quirúrgica usada para el tratamiento definitivo de la uña encarnada.

Objetivos. Los objetivos del estudio fueron: 1.- Cuantificar el tiempo medio de curación de los pacientes de la muestra sometidos a una matricectomía parcial del la uña por técnica fenol alcohol y 2.- Calcular la frecuencia de aparición de las principales complicaciones de éste procedimiento en nuestra muestra.

Métodos. Se seleccionó una muestra de 44 pacientes intervenidos de uña encarnada durante el período de tiempo comprendido entre el 1 de enero del 2007 y el 1 de enero del 2012, y se calculó el tiempo medio de curación. El siguiente paso fue realizar unas tablas con las complicaciones que aparecieron entre los 0-60 días, y entre los 61-365 días, y se calculó la frecuencia de aparición de cada una de ellas en nuestra muestra.

Resultados. Se obtuvo que el tiempo medio de curación fue de 30,4 días. Las complicaciones fueron escasas, siendo el seroma una de las principales complicaciones (6,81\%). Destacar el bajo índice de recidivas que se obtuvo: $0 \%$ recidiva total y $4,54 \%$ de recidiva parcial.

Conclusiones. Es una técnica muy estudiada y controvertida, que ha sufrido numerosas variaciones. La realización de la técnica tal y como la describimos en nuestro estudio nos proporciona unos resultados muy satisfactorios, con un bajo índice de complicaciones y recidivas. Por ello podemos concluir que es una técnica válida y eficaz para el tratamiento de la uña encarnada.

Palabras clave: uña encarnada; cirugía; phenol-alcohol; complicaciones.

\footnotetext{
ABSTRACT

Original study.

Introduction. The phenol alcohol chemical matricectomy is a surgical technique used for the definitive treatment of ingrown toenail.

Aims. The objectives of the study were: 1.- Quantify the average healing time of patients in the sample subjected to matricectomy the nail art of phenol alcohol and 2. - Calculate the frequency of major complications of this procedure in our sample.
}

Methods. To a sample of 44 patients for undergoing ingrown toenail from January 1, 2007 to January 1, 2012, and 
calculated the average healing time. The next step was to hold a draw with the complications that appeared between 0-60 days, and between 61-365 days, and calculated the frequency of occurrence of each of them in our sample.

Results. - It was found that the average healing time was days. The complications were rare, being seroma the major complication (6,81\%). Highlighting the low rate of recurrence rate was obtained: $0 \%$ of total recurrence and of $4.54 \%$ partial recurrence.

Conclusions. - Is a technique widely studied and controversial, it has undergone many changes. The performance of the technique as we described in our study provides very satisfactory results, with a low rate of complications and recurrences. Therefore we can conclude that it is a valid and effective for the treatment of ingrown toenail.

Keywords: ingrown toenail; surgery; phenol-alcohol; complications.

Sumario: 1.Introducción. 2. Materialy Métodos. 3. Resultados.4.Resultados. 5.Discusión. 6. Conclusiones. Bibliografía.

Referencia normalizada: Lasanta Lacalzada, L., Reyes Martín, R. Técnica fenol alcohol para el tratamiento de uñas encarnadas. Tiempo medio de curación y complicaciones para una muestra de mayores de 65 años. Rev. Int. Cienc. Podol. 2015; 9(2): 74-84. 


\section{INTRODUCCIÓN}

La onicocriptosis o uña encarnada es uno de los motivos más frecuentes en consulta de podología. Se produce cuando la lámina ungueal penetra en los tejidos blandos que la rodean produciendo la inflamación de los mismos e incluso la infección ${ }^{1}$.

Esta situación se puede producir por elementos extrínsecos como presión excesiva del calzado, corte incorrecto de las uñas, onicofagia, traumatismos; o por causas intrínsecas: lecho ungueal ancho y grosor de la lámina ungueal fino, neoplasias subungueales, hiperhidrosis, alteraciones biomecánicas, o combinaciones de ambas ${ }^{1}$.

Según la gravedad de la onicocriptosis se han descrito cuatro estadíos. El estadío I que cursa con edema, eritema y dolor. Estadío II en el cual aparece la infección y la herida empieza a drenar. Estadío III ${ }^{2,3}$. Surge el tejido de granulación y la hipertrofia lateral de las paredes y el estadío IV, descrito por Martinez Nova et $\mathrm{al}^{4}$, caracterizado por hipertrofia tanto de los pliegues laterales como del pliegue distal. Este sería el estado clínico más grave de esta enfermedad.

Como tratamiento definitivo a dicho problema, la literatura propone distintos tipos de cirugía ungueal. La cirugía se indica de manera electiva en los estadíos III y IV, en las onicocriptosis recidivantes (en cualquier estadío), en las recidivas quirúrgicas o cuando el tratamiento conservador falla ${ }^{4}$.

Para abordar el tratamiento quirúrgico de la uña encarnada podemos optar por matricectomías incisonales o por matricectomías químicas mediante el empleo de sustancias químicas para conseguir la erradicación de la matriz ungueal. La literatura médica coincide mayoritariamente en recomendar la matricectomía química con fenol sobre matricectomías quirúrgicas incisionales ${ }^{5,6}$ la considera técnica de elección en los casos que no necesitamos obtener muestra histológica ${ }^{2,7,8}$.

En nuestra práctica habitual la técnica más usada es la matricectomía parcial de la uña con técnica fenol-alcohol. Consiste en la exéresis de la espícula ungueal afecta y una matricectomía parcial mediante quemadura química por aplicación de fenol.
El fenol produce una quemadura concentrada de tercer grado del tejido matricial. Durante varias semanas supura un exudado seroso a medida que la herida se desprende del tejido necrótico y alcanza su curación.

La cirugía ungueal por técnica fenol alcohol fue descrita por primera vez por Otto Boll en 1943. Posteriormente han sido otros autores los que han ido proponiendo variaciones a dicha técnica, fundamentalmente en lo relativo a los tiempos de aplicación del fenol y número de fenolizaciones ${ }^{9,10,11,12,13}$.

Hemos encontrado numerosos artículos en los que se describen los pasos a seguir al realizar está técnica, las complicaciones más frecuentes, y comparativas de resultados con la realización de otras técnicas ${ }^{1,8,14,15,16,17}$. Se la suele describir como una técnica eficaz pero con un largo periodo de curación, por ello en este estudio, queremos cuantificar realmente cuales son los tiempos medios de curación que tenemos en nuestra práctica clínica.

Así mismo nos hemos centrado en revisar las posibles complicaciones que aparecen descritas en la bibliografía respecto al postoperatorio de esta técnica. Dichas complicaciones aparecen bien descritas pero no hemos encontrado en la bibliografía con que frecuencia aparecen, por ello queremos valorar el índice de complicaciones que tenemos tras la realización de esta cirugía en nuestra práctica habitual y cuáles son las complicaciones más y menos frecuentes.

\section{OBJETIVOS}

Al ser un estudio descriptivo no planteamos hipótesis y si no los objetivos de la investigación que serán:

- Cuantificar el tiempo medio de curación de los pacientes, con un rango de edad mayores de 65 años sometidos a una matricectomía parcial de la uña del primer dedo por técnica fenol-alcohol en nuestra consulta de podología en los últimos 5 años. (Del 1 de enero 2007 al 1 de enero 2012).

- Calcular la frecuencia de aparición de las principales complicaciones de éste procedimiento en nuestra muestra. 


\section{MATERIAL Y MÉTODOS}

Se realizó un estudio de carácter Observacional Descriptivo Transversal Retrospectivo.

El trabajo tuvo lugar en la Clínica del Pie, sita en la calle Camino de Leganés 177, Madrid y se basó en el estudio a través de las historias clínicas de una muestra de 44 pacientes, hombres y mujeres, con una edad comprendida entre los 66 y los 86 años. Todos ellos acudieron a consulta de podología por presentar una uña encarnada en la uña del primer dedo del pie, en uno o ambos canales ungueales, y se decidió su intervención por el grado de recurrencia del proceso o por su grado de afectación.

Todos los pacientes de la muestra presentaron una uña encarnada del primer dedo y como tratamiento definitivo se les realizó una matricectomía parcial, unilateral o bilateral, de la uña por técnica fenol alcohol, durante un periodo de tiempo comprendido entre 1 de enero del 2007 al 1 de enero del 2012.

- Para ser incluidos en el estudio a todos los pacientes se les solicitó analítica previa a la cirugía (bioquímica, hemograma y coagulación) y se realizó proyección radiológica lateral y anteroposterior del hallux.

- Como criterios de exclusión del estudio:

- Pacientes fuera de rango de edad.

- Pacientes con parámetros alterados en la analítica.

- Pacientes inmunodeprimidos.

- Enfermedad arterial severa.

- Presencia de exóstosis subungueal, o tumores en la falange distal del primer dedo.

- Presencia de osteomielitis.

- Pacientes con afectación ungueal tipo IV, según clasificación de Martinez Nova et al. ${ }^{4}$

- Alteraciones morfofuncionales que sometan al hallux a solicitaciones mecánicas excesivas en cualquiera de sus planos, como hallux flexus, hallux extensus y alteraciones rotacionales del hallux.

- La necesidad de recoger muestra para estudio anatomopatológico.

- De las historias clínicas se recogieron las variables edad, sexo, fecha de realización de la cirugía, fecha de alta, tiempo transcu- rrido entre ambas fechas, complicaciones y recidivas.

- Paciente con estadios I no se les pautó antibioterapia oral. Y en estadíos II y III sí.

\section{Descripción de la intervención}

A todos los pacientes intervenidos se les anestesió con mepivacaina al $2 \%$ svc. Una vez comprobada la anestesia, ya con técnica estéril, se realizó isquemia con venda $S$ march $1,5 \mathrm{~cm}$ de ancho.

Comenzamos con un alicate inglés realizando un corte recto de la uña de distal a proximal evitando la divergencia del corte; aproximadamente a $2-3 \mathrm{~mm}$ del borde clavado, justo donde comienza la convexidad ungueal. Seguidamente continuamos el corte hasta la raíz de la uña con una gubia o escoplo, teniendo especial cuidado de no dañar el eponiquio. Después realizamos la avulsión del fragmento ungueal, empleando un mosquito recto, que lo vamos a clampar en la zona más proximal a la cutícula, realizando un giro en sentido contrario al del lado enclavado.

Una vez extraído el fragmento con una cucharilla de Wolkman realizamos un ligero legrado de la zona de proximal a distal para verificar que no queda ningún fragmento. Debemos legrar en tres sentidos dorsal, plantar y lateral, nunca en sentido de la uña sana porque puede penetrar fenol y lesionar zona sana. En ese momento procedemos a limpiar el canal ungueal, comprobando que no quede ningún resto sérico.

En estadío III y IV se retira tejido de granulación y se remodela la hipertrofia de los bordes, con un bisturí del número 15 .

Dependiendo del tamaño del canal ungueal vamos a personalizar con algodón estéril el tamaño de las torundas que vamos a utilizar para la fenolización. Impregnamos la torunda de algodón en fenol, retiramos el exceso con una gasa e introducimos en la matriz ungueal y canal ungueal.

Utilizamos fenol licuado al $89 \%$ y realizamos dos fenolizaciones de 45 segundos sin lavar con alcohol entre una y otra. Irrigando con abundante alcohol, con jeringa de 10cc al finalizar la segunda fenolización.

Finalizada la fenolización y la irrigación con alcohol, secamos la zona, aplicamos povidona 
yodada al 10\% y aplicamos mupirocina tópica. Cubrimos con apósito antiadherente y vendamos el dedo.

Seguidamente mantenemos al paciente durante 15-20 min con el pie elevado. Administramos analgesia vía oral, ibuprofeno 400$600 \mathrm{mg}$ como primera elección, y en caso de estar contraindicado valoramos alternativa. Habitualmente con el comprimido administrado al finalizar la cirugía suele ser suficiente para mitigar el dolor, no obstante siempre referenciamos al paciente la posibilidad de continuar la dosis cada $8 \mathrm{~h}$ si el dolor persiste.

La primera revisión se realiza a las 24 horas: retiramos vendaje, lavado con suero fisiológico, aseptización de la zona con povidona yodada $10 \%$ y aplicación de mupirocina.

El paciente realizará curas diarias domiciliarias con lavado con suero salino en inmersión del pie durante 3-5 min. Abriendo canal ungueal y realizando técnica de arrastre con una gasa de proximal a distal. Aseptización de la zona con povidona yodada $10 \%$ y aplicación de mupirocina $2 \%$. Hasta completar 10 días.

A los 7 días de la intervención se revisa en consulta y se procede a retirar tejido de granulación.

A partir de los 10-12 días suspendemos los lavados en inmersión y la aplicación de mupirocina al 2\%, recomendando la aplicación únicamente de povidona yodada al $10 \%$. Curas en consulta 1 vez por semana hasta su total curación.

\section{Análisis de los datos}

En el estudio se realizó una tabla en la que se recogió el tiempo medio de curación en pa- cientes sometidos a matricectomía parcial por técnica fenol alcohol. Dicho tiempo se caculó desde la fecha de la intervención hasta el alta podológica, que se dió el día en que el canal ungueal había epitelizado en su totalidad. Así mismo se hizo un desglose por sexos del tiempo medio de curación.

Posteriormente se elaboraron unas tablas con las complicaciones que aparecen entre 0-60 días y entre los 61-365, y describimos en que porcentaje nos han aparecido en la muestra.

Los datos recogidos se informatizaron mediante una hoja de cálculo Excel, por medio de la cual se realizaron las tablas que aparecen en el estudio. Utilizamos la media muestral para calcular la media de edad de la muestra a estudio y para calcular el tiempo medio de curación.

\section{Consideraciones éticas}

A todos los pacientes se les solicitó, por escrito, la firma del consentimiento informado. Se les dio a leer un documento en el que se describía la técnica que se les iba a realizar y sus posibles complicaciones. Todos los pacientes lo firmaron.

\section{RESULTADOS}

Se registraron 44 casos de pacientes con uña encarnada del primer dedo que fueron sometidos a una cirugía ungueal por técnica fenol alcohol, de uno o ambos canales ungueales.

Los pacientes intervenidos tenían una edad comprendida entre los 66 y los 86 años, con una edad media de 72,2 años.

\section{Muestra $\mathrm{N}=44$}

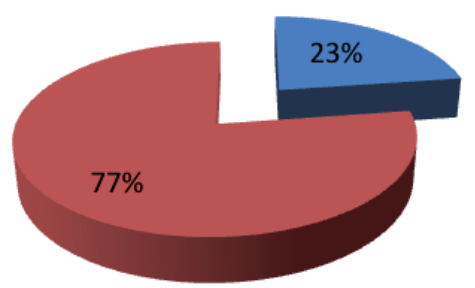

Gráfico 1. Muestra por sexos. 


\section{Tiempo de curación}

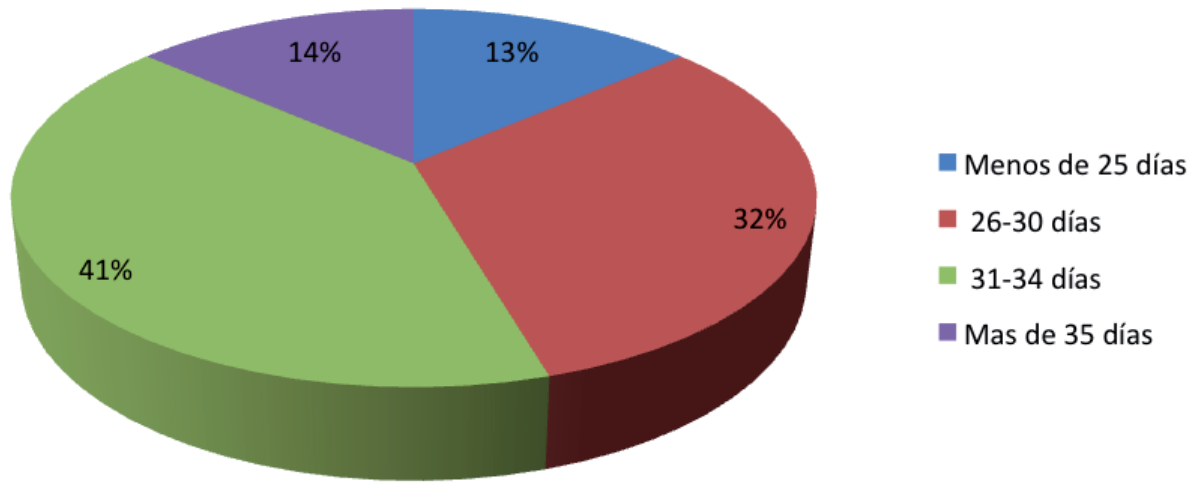

Gráfico 2. Tiempo de curación.

El $77 \%$ fueron mujeres (34 casos) y el $23 \%$ hombres (10 casos) (Gráfico 1).

El tiempo medio de curación estuvo en los días 30,4 días.

Desglosamos este período de curación por sexos y obtuvimos que:

- Tiempo medio de curación en mujeres fue de 31,2 días.

- Tiempo medio de curación en hombres fue de 27,6 días.

Del total de la muestra nos encontramos que: (Gráfico 2).

- Un $13 \%$ de los pacientes sometidos al estudio tardaron 25 o menos días en obtener el alta.

- Un $32 \%$ de los pacientes tardaron entre 26 y 30 días.

- Un $41 \%$ tardaron entre 31 y 34 días.

- Un 14\% tardaron 35 o más días en obtener el alta.

Los 44 pacientes realizaron una vida normal a partir de las 48 horas.

Para recopilar las complicaciones en la técnica de fenol alcohol se realizó una revisión bibliográfica ${ }^{1,14,18,19,20,21}$, extrayendo las principales y posteriormente clasificándolas en dos grupos según el tiempo de aparición. Además se calculó la frecuencia de aparición de dichas complicaciones en nuestra muestra.
1. Complicaciones que se produjeron entre los días 0-60 días:

1-1. Complicaciones comunes a cualquier cirugía Trauma por inyección del anestésico.

- Trauma por la aplicación del torniquete.

- Hemorragia.

- Dolor.

- Infección.

1-2.Complicaciones propias a la técnica fenol alcohol

- Quemadura por fenol.

- Periostitis.

- Formación de seromas.

2. Complicaciones que se producen entre los 61-365 días

Afectación del crecimiento de la uña:

- Pérdida total de uña.

- Crecimiento ectópico de la uña.

- Lateralización de la uña.

- Recidiva parcial (espícula).

- Recidiva total.

En el grupo 1-1 de complicaciones de una muestra $\mathrm{N}=44$ el $93,18 \%$ no tuvo ningún tipo de complicación. La frecuencia de aparición de las complicaciones secundarias al trauma de inyección y del torniquete fue del $0 \%$. La hemorragia tuvo una frecuencia de aparición del $2,27 \%$; el dolor del $2,27 \%$ y la infección del 2,27\% (Gráfico 3). 
Del grupo 1-2 de complicaciones propias en el desarrollo de la técnica de fenol alcohol, se destacó la ausencia de complicaciones tanto de quemaduras por fenol como de periostitis. Existió una frecuencia de aparición de seromas del 6,81\% (Gráfico 4).

Las complicaciones del grupo 2 son aquellas que van a afectar al crecimiento de la uña una vez realizada la técnica y transcurridos al menos 61días. El 93,18\% de la muestra no presentaron complicaciones. La frecuencia de aparición de crecimiento ectópico de la uña y lateralización de la uña fue del $0 \%$. Un 2,27\% tuvo una pérdida de la uña en el transcurso de este período; un $4,54 \%$ tuvo una recidiva parcial (espícula), que no necesitaron reintervención posterior; y no tuvimos ningún caso de recidiva total (Gráfico 5).

\section{1-1 Complicaciones entre 0-60 días}

\begin{tabular}{cccccc}
\multicolumn{7}{c}{ Complicaciones comunes entre 0-60 días } \\
\hline 41 & 0 & 0 & 1 & 1 & 1 \\
\hline $\begin{array}{c}\text { Sin } \\
\text { complicaciones } \\
93.18 \%\end{array}$ & $\begin{array}{c}\text { Trauma } \\
\text { inyección } 0 \%\end{array}$ & $\begin{array}{c}\text { Trauma } \\
\text { torniquete } 0 \%\end{array}$ & $\begin{array}{c}\text { Hemorragia } \\
2.27 \%\end{array}$ & Dolor 2.27\% & $\begin{array}{c}\text { Infección } 2.27 \% \\
\%\end{array}$ \\
\end{tabular}

Gráfico 3. Complicaciones entre 0-60 días.

\section{1-2 Complicaciones entre 0-60días}

- Complicaciones propias de la técnicas entre 0-60 días.

\begin{tabular}{cccc}
41 & 0 & 0 & 3 \\
\hline $\begin{array}{c}\text { Sin complicaciones } \\
93,18 \%\end{array}$ & $\begin{array}{c}\text { Quemaduras por fenol } \\
0 \%\end{array}$ & Periostitis $0 \%$ & Seromas 6,81\%
\end{tabular}

Gráfico 4. Complicaiones entre 0-60 días.

\section{Complicaciones entre 61 -365 días}

- Complicaciones que afectan al crecimiento de la uña.

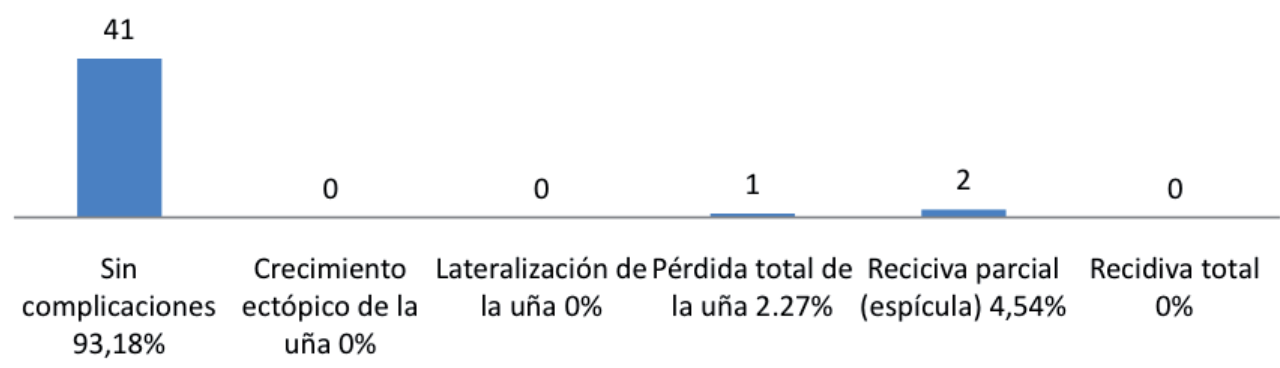

Gráfico 5. Complicaciones entre 61-365 días. 


\section{DISCUSIÓN}

La técnica fenol alcohol es una de las técnicas quirúrgicas ungueales más utilizadas en podología. Al mismo tiempo es una de las más discutidas, ya que son muchos los estudios que han aportado ajustes y variaciones a su descripción original.

Dadas las propiedades anestésicas y antisépticas del fenol, el dolor y las infecciones postquirúgicas son poco frecuentes y el resultado funcional y estético es excelente.

Es una técnica que nos ofrece múltiples ventajas:

- Índice de recidiva bajo, entre 1,1\% y $11 \%$. $7,22,23,24,25$ (en nuestro estudio: $0 \%$ recidiva total y $4,54 \%$ recidiva parcial).

- Menores requerimientos de analgésico postoperatorio que en técnicas incisionales $^{26}$.

- Posibilidad de realizar en presencia de infección ${ }^{27,28}$ e incluso en pacientes diabéti$\cos ^{24}$.

El principal inconveniente de la técnica es un período postquirúrgico prolongado, de alrededor de las 4 semanas. Coinciden los resultados de nuestro estudio (30,4 días) con el tiempo señalado por otros autores. ${ }^{1,14,18}$. Aunque en el desarrollo del postoperatorio el paciente se reincorpora a sus actividades cotidianas a partir de las 24-48h.

La posibilidad de sufrir complicaciones va a venir determinada por causas intrínsecas al paciente (obesidad, diabetes, malnutrición, edad avanzada, inmunosupresión) o por causas derivadas de una mala praxis del podólogo tanto en la preparación como en la ejecución del acto quirúrgico, o dado que es un procedimiento ambulatorio, por un incumplimiento de las recomendaciones posquirúrgicas o por unos hábitos higiénicos inadecuados. De esta forma, nos encontramos en nuestro estudio que la complicación más frecuente es la aparición de seromas $(6,81 \%)$ íntimamente ligado a una inadecuada realización de las curas domiciliarias. En ocasiones nos encontramos con pacientes aprensivos o poco disciplinados, en esos casos recomendamos aumentar la frecuencia de las visitas en consulta para la realización de la cura por un profesional.
Es por ello que el podólogo debe elegir perfectamente el candidato susceptible de dicha cirugía, debe dominar perfectamente la técnica operatoria, debe informar al paciente de su compromiso a la hora de realizar sus curas domiciliarias manteniendo la zona intervenida en un ambiente higiénico óptimo, y debe reconocer a la mayor brevedad posible el inicio de complicaciones si las hubiese.No obstante, considerada esta técnica una de las más aceptadas es una de las más discutidas. Existe mucha controversia en relación con la aplicación del fenol en tiempo, concentración y número de aplicaciones. Así como la necesidad de irrigar o no con alcohol después de cada fenolización. La concentración del fenol aplicado varía entre el 80 y el $100 \%{ }^{9}$ Butterworth y Dockery aplican fenol al $100 \%$ en dos tiempos, cada una de un minuto, y no emplean alcohol, porque consideran que no produce efecto alguno. Yale 10,11.aplica fenol al $88 \%$ en dos tiempos, de 1,5 y 3 minutos, y Giacolone ${ }^{12}$. lo hace al $89 \%$ en tres tiempos de 0,5 minutos; ambos irrigan sólo una vez con alcohol, al final de las aplicaciones de fenol. Otros autores, como González Díaz y cols ${ }^{13}$ se guían por el cambio al color blanco de la zona matricial al aplicar el fenol, sin establecer un tiempo en concreto. Grennwald y Robins ${ }^{29}$ opinan que el alcohol produce una dilución y no neutraliza, lo que disminuye el poder cáustico del fenol. Jeffrey $\mathrm{S}$ Bober y cols $^{30}$ demostraron que la concentración necesaria para la destrucción completa de la uña es del $89 \%$, y precisa de un tiempo mínimo de un minuto. Por ello, aplican el fenol en dos tiempos de 30 segundos, con irrigación de alcohol después de cada aplicación.

En nuestra opinión, utilizando un menor tiempo de exposición de fenol en la matriz ungueal disminuimos los riesgos de tener complicaciones tales como periostitis, quemaduras. En nuestro estudio, aplicando dos fenolizaciones de 45 segundos de fenol licuado al 89\% hemos obtenido un índice de recidiva total del $0 \%$, y de recidiva parcial del $4,54 \%$,considerando innecesario realizar un mayor tiempo del fenolizaciones, ya que estamos en el rango inferior del número de recidivas de que describe en la bibliografía $(1,1 \%-11 \%)^{7,22,23,24,25}$.

Para evitar complicaciones derivadas del uso del fenol nos decantamos por personalizar 
el tamaño de las torundas de algodón ésteril según la cantidad de uña extirpada. Aplicando la cantidad justa de fenol, retirando el sobrante, para evitar que se extravase y produzca quemaduras adyacentes. Preferimos esta opción sobre otros métodos de aplicación descritos (hemostetas, bastoncillos) ${ }^{1,18}$; y sobre la práctica que señalan algunos autores de proteger la zona eponiquial con vaselina ${ }^{1}$, ya que existe el riesgo de ésta penetre en la zona matricial y que el fenol no pueda producir la quemadura completa de la matriz, con el consiguiente riesgo de recidiva.

Para evitar el riesgo de producir periostitis ${ }^{19}$ debemos no ser demasiado enérgicos en el legrado vigilando que a la hora de extraer el fragmento ungueal no se hay producido ninguna laceración que conduzca al fenol al tejido óseo.

Par finalizar, dejamos abierta la puerta a futuros estudios que relacionen y cuantifiquen la fre- cuencia de aparición de complicaciones y el índice de recidivas, con los distintos tiempos, número de fenolizaciones y concentración del fenol.

\section{CONCLUSIONES}

La onicocriptosis es una afección recurrente y dolorosa, que debe ser subsidiaria de un procedimiento quirúrgico sencillo y rápido, con buenos resultados funcionales y estéticos, que permitan al paciente una reincorporación rápida a sus actividades y que tenga un índice de recidivas bajo.

La técnica de fenol-alcohol puede ser una buena alternativa, es una técnica rápida, sencilla y segura Es una técnica poco invalidante para el paciente, con un bajo índice de complicaciones. Y dado el bajo índice de recidivas, la consideramos válida y eficaz.

\section{BIBLIOGRAFÍA}

1. Fernández-Jorge B, Peña Penabad C, García-Silva J. Tratamiento de la onicocripsis con matricectomía química con fenol. Piel. 2009; 24(1): 46-51.

2. Bostanci S, Ekmekci P, Gürgey E. Chemical matricectomy with phenol for the treatment of ingrowing toenail: a review of the literature and follow-up of 172 treated patients. Acta Derm Venereol. 2001; 81:181-3.

3. Siegle RJ, Stewart R. Recalcitrant ingrowing nails. J Dermatol Surg Oncol. 1992; 18:744-52.

4. Martínez-Nova A, Sánchez- Rodriguez R, Alonso-Peña D. New onychocrytosis classification and treatment plan. J Am Podiatr Med Assoc. 2007; 97: 389-93.

5. Cameron PF. Ingrowing toenails: an evaluation of two treatments. Br Med J (Clin Res ED) 1981; 283:821-2.

6. Bos AM, Van Tilburg MW, Van Sorge AA, Klinkenbijl JH. Randomized clinical trial of surgical technique and local antibiotics for ingrowing toenail. Br J Surg.

7. Kimata Y, Uetake M, Tsukada S, Harii K. Follow-up study of patients treated for ingrown nails with the nail matrix phenolization method. Plast Reconstr Surg.1995;95:719-24.

8. Islam S, Lin EM, Drongowski R, Teitelbaum DH, Coran AG, Geiger JD, et al. The effect of phenol on ingrown townail excision in children. J Pediatr Surg. 2005; 40:290-2.

9. Butterwort R, Dockery GL. Atlas a color y texto de cirugía del antepié. Madrid: Ortocen, 1992; 73-74.

10. Yale JF. Special techniques in nail surgery. En: Jay RM, ed. Current therapy in podiatric surgery. Toronto: Decker, 1989; 115.

11. Yale JF. Phenol-alcohol technique for correction of infected grown toenail. J Am Podiatry Assoc 1974; 64:46.

12. Giacalone VF. Phenol matricectomy in patient with diabetes. J Foot Ankle Surg 1997; 36: 264-267.

13. González Díaz JC, Pascual Huerta J, Ropa Moreno JM, García Carmona FJ, Moreno de Castro M, Lázaro Martínez JL. Revisión bibliográfica de la técnica fenol alcohol en el tratamiento de la onicocriptosis. Rev Esp Podol 1999; 10: 42-65.

14. De Felipe Medina R, Andrade Rosa C, Cruz Quintás L, Alfonso Camús J, Alfageme Lastra MS. Cirugía de la uña en atención primaria. Estudio de casos intervenidos en un centro de atención primaria en los últimos 3 años. Originales SEMG. 2007. Disponible en: http//www.medicinageneral.org/revista.95/pdf/220-222.pdf.

15. Espensen E. Nixon BP, Armstrong DG. Chemical matrixectomy for ingrown toenails. I there an evidence basis to guide therapy. J Am Podiatr Med Assoc 2002; 92 (5):287-295. 
16. Boberg JS, Frederiksen MS, Harton FM. Scientific analysis of phenol nail surgery. J Am Podiatr Med Assoc 2202; 92(10): 575-579.

17. Farley-Sakevich T, Grandy JF, Zager EM, Axe TM. Onychoplasty with carbon dioxide laser matrixectomy for treatment of ingrown toenails. J Am Podiatr Med Assoc 2005; 92(2): 175-179.

18. Martinez Franco A. Matricectomía química parcial. Técnica de fenol-alcohol. En: Martinez Nova A. Podología.Atlas de cirugía ungueal. Madrid: Panamericana; 2006.p.111-125.

19. Piña Delgado G., García Carmona F.J. Complicaciones en cirugía ungueal. En: García Carmona F.J. Patología y cirugía ungueal. Barcelona: Mayo; 2008.p.79-91.

20. Novel I Marti V. Cirugía ungueal. En: Izquierdo Cases O. Podología Quirúrgica. Madrid: Elsevier; 2006.p.41-53.

21. Jenkin,W. Cirugía de la uña: avulsiones y matricectomías ungueales. En: Chang T. Técnicas en cirugía ortopédica. Pie y tobillo. Madrid: Marbán; 2006.p.1-13.

22. Morkane AJ, Robertson RW, Inglis GC. Segmental phenolization of ingrowing toenails: a randomized controlled study.Br J Surg. 1984; 71: 526-7.

23. Andreassi A, Grimaldi L, D’Aniello C, Pianigiani E, Bilenchi R. Segmental phenolization for the treatment of ingrowing toenails: a review of 6 years experience. J Dermatolog Treat. 2004; 15: 179-81.

24. Giacalone VF. Phenol matricetomy in patients with diabetes. J Foot Ankle Surg. 1997; 36: 264-7.

25. Byrne DS, Caldwell D. Phenol cauterization for ingrowing toenails: a review of five years' experience. Br J Surg. 1989; 76:598-9.

26. De Berker DA. Phenolic ablation of the nail matrix. Australas J Dermatol. 2001; 42: 59-61.

27. Van der Ham AC, Hackeng CA, Yo TI. The treatment of ingrowing toenails. A randomized comparison of wedge excision and phenol cauterization. J Bone Joint Surg Br. 1990; 72:507-9.

28. Barn R, Haneke E. Matricectomy and nail ablation. Hand Clin. 2002:18; 693-6.29.- Grenwald L, Robbins HM. The chemical matricectomy: a commentary. J Am Podiatry Assoc 1981; 71:388.

30. Boberg JS, Frederiksen MS, Harton FM. Scientific analysis of phenol nail surgery. J Am Podiatry Med Assoc 2002; 92: 575-579. 